\title{
Intraoperative hypertension in a patient with undiagnosed pheochromocytoma under spinal anesthesia
}

\author{
Youn Hee Lim, Won Ji Rhee, So Ron Choi, Sang Won Park, and Chan Jong Chung \\ Department of Anesthesiology and Pain Medicine, College of Medicine, Dong-A University, Busan, Korea
}

Pheochromocytoma is a catecholamine-secreting tumor. Reduction of the high risk of intraoperative hypertension during tumor manipulation requires adequate preoperative preparation. Even though adequate pretreatment is performed, uncontrolled hemodynamic responses during the surgery may result in postoperative complications. In particular, undiagnosed pheochromocytoma without any pretreatment can lead to highly severe life-threatening hypertensive crisis [1]. Therefore, it has always posed a great challenge for anesthesiologists [2]. In the case of our patient, induction of spinal anesthesia helped in the prevention of severe hypertension and, consequently, the development of severe complications.

A 45-year-old woman, $153 \mathrm{~cm}$ in height, weighing $63 \mathrm{~kg}$, was scheduled to undergo transurethral resection of a bladder tumor (TUR-BT) because of a suspected hemangioma. She had a 5-year history of dysuria. Cystoscopy showed a single nodular mass (approximately $2.5 \mathrm{~cm}$ ) on the anterior wall of the bladder. Contrast-enhanced computed tomography showed a pedunculated and well-enhanced mass lesion.

Findings of preoperative laboratory examinations were within the normal limits. The preoperative blood pressure (BP) was $110 / 60 \mathrm{mmHg}$ and the heart rate (HR) was 70 beats/min (bpm). The patient had no medical illnesses. Two years ago, she had undergone laparoscopic cholecystectomy under general anesthesia uneventfully.

In the operating room, an EKG, a non-invasive BP measuring instrument, and a pulse oximeter were set up for monitoring the patient. Using a 25-gauge Quincke needle, we administered the patient $13 \mathrm{mg}$ of $0.5 \%$ heavy bupivacaine and $20 \mu \mathrm{g}$ fentanyl via intrathecal injection. A sensory block to T6 was confirmed 5 minutes later. Subsequently, intravenous injection of $2 \mathrm{mg}$ of the sedative midazolam was administered. The patient remained hemodynamically stable for $40 \mathrm{~min}$ until tumor manipulation, when, the BP and HR increased from 100/50 to 220/120 mmHg and from 50 to $70 \mathrm{bpm}$, respectively. Simultaneous EKG showed ventricular bigeminy. Immediately after $60 \mathrm{mg}$ of lidocaine was intravenously administered, the arrhythmia disappeared. However, on recovery from sedation, she complained of headache and chest discomfort without any pain at the site of operation. Thus, she was intravenously administered $80 \mu \mathrm{g}$ of fentanyl and $2 \mathrm{mg}$ of midazolam.

Subsequently, her BP decreased to $180 / 100 \mathrm{mmHg}$, but she was not sedated. This allowed us to obtain additional details of her medical history. She stated that for the past 9 years, she had occasionally experienced sudden intense headache and chest tightness immediately after urination. Neurologic and cardiologic examinations were performed, but no specific findings were obtained.

The patient was sedated with an intravenous infusion of propofol and fentanyl. The systolic BP continued to fluctuate from 130 to $150 \mathrm{mmHg}$; however, a hypertensive crisis, which has been previously observed during tumor manipulations, did not occur in this patient. After a discussion with the operator, we collected a sample of the tumor mass for biopsy and completed the surgery. Immunohistochemical analysis showed that the tumor was a paraganglioma (pheochromocytoma) with a high malignant potential. The postoperative phase was uneventful.

Corresponding author: So Ron Choi, M.D., Ph.D., Department of Anesthesiology and Pain Medicine, College of Medicine, Dong-A University, Dongdaeshindong 3-ga, Seo-gu, Busan 602-715, Korea. Tel: 82-51-240-5390, Fax: 82-51-247-7819, E-mail: choisr@dau.ac.kr

(c) This is an open-access article distributed under the terms of the Creative Commons Attribution Non-Commercial License (http:// creativecommons.org/licenses/by-nc/3.0/), which permits unrestricted non-commercial use, distribution, and reproduction in any medium, provided the original work is properly cited. 
For 20 days, the patient was administered a daily oral dose of $4 \mathrm{mg}$ doxazosin. According to the Roizen criteria, the patient did not show severe postural hypotension, headache, or chest tightness during hospitalization [2]. A partial cystectomy was planned. The initial BP was $117 / 70 \mathrm{mmHg}$ and $\mathrm{HR}$ was $75 \mathrm{bpm}$. Radial arterial cannula was inserted for invasive BP monitoring. Hydration was maintained using intravenous fluids. To avoid severe hypertension during the induction period, we intravenously injected $50 \mu \mathrm{g}$ fentanyl and $60 \mathrm{mg}$ lidocaine. Anesthesia was induced with intravenous injections of $120 \mathrm{mg}$ propofol and $50 \mathrm{mg}$ rocuronium. The trachea was gently intubated with a cuffed endotracheal tube. No significant hemodynamic disturbances were observed during anesthetic induction. Anesthesia was maintained with 1.5$2.5 \%$ sevoflurane and $50 \%$ nitrous oxide in oxygen. The BP did not significantly increase during the operation. After the remnant pedunculated mass was clamped, the BP decreased from $100 / 50$ to $70 / 40 \mathrm{mmHg}$, and the HR increased from 65 to $90 \mathrm{bpm}$, without any significant bleeding. Initially, intravenous fluid administered, followed by continuous dopamine (3-6 $\mu \mathrm{g} / \mathrm{kg} / \mathrm{min}$ ) and phenylephrine $(0.5-1 \mu \mathrm{g} / \mathrm{kg} / \mathrm{min})$ infusions [3]. Thereafter, her vital signs remained stable until the end of the surgery. Her fluid intake included 2,100 ml of crystalloid and $500 \mathrm{ml}$ of colloid; her urine output was $1,200 \mathrm{ml}$ and blood loss was $100 \mathrm{ml}$. Dopamine infusion was discontinued after she was transferred to the postanesthesia care unit because she remained hemodynamically stable. She was shifted to the general ward and was uneventfully discharged after 7 days.

In our case, the patient underwent surgery twice. Fortunately, the first operation was performed under spinal anesthesia, which serves as systemic sympathetic blockade. Therefore, severe hypertensive crisis did not occur. Moreover, because of the patient's consciousness, pheochromocytoma could be suspected at an early stage, and this might contribute to her postoperative stabilization. During the second operation, the patient remained stable owing to the appropriate preoperative preparations and the small size of the remnant mass. The patient recovered from the disease without any postoperative complications.

In summary, a patient presenting with unexplained hypertension and tachycardia, especially associated with tumor manipulation need to be examined for pheochromocytoma. If undiagnosed pheochromocytoma is suspected during operation, surgical manipulation should be interrupted and appropriate medical treatment should be commenced.

\section{References}

1. Kim KB, Chang CH, Shin CS, Lee JW, Nam SB. Unilateral pulmonary edema during an operation in patient with undiagnosed pheochromocytoma. Korean J Anesthesiol 2008; 55: 502-5.

2. Myklejord DJ. Undiagnosed Pheochromocytoma: The Anesthesiologist Nightmare. Clin Med Res 2004; 2: 59-62.

3. Desmonts JM, Marty J. Anesthetic management of patients with pheochromocytoma. Br J Anaesth 1984; 56: 781-9. 